\title{
DEPRESSÃO PÓS-PARTO: UM ENFOQUE À SAÚDE MENTAL DA PUÉRPERA SOB A PERSPECTIVA DA ENFERMAGEM
}

\author{
Andrea Almeida Zamorano ${ }^{1}$
}

RESUMO: Este artigo ressalta a importância temática baseada no enfoque à saúde mental da puérpera e as contribuições do enfermeiro no reconhecimento e intervenções através do diagnóstico precoce da DPP como forma de amenizar o sofrimento psíquico da parturiente e repercussões da dor psíquica na vida social e familiar. Relata a importância do pré-natal intermediado por alterações que podem acarretar à depressão pós-parto. O presente estudo busca esclarecer a etiologia, a importância do diagnóstico precoce, suas variadas formas de tratamento e ações preventivas para a depressão pós-parto do qual são decorrentes de alterações emocionais, cognitivas, comportamentais e físicas desencadeadas do início gestacional até a fase puerperal. Por meio de uma revisão bibliográfica e sistemática da literatura, observamos que sua etiologia não foi totalmente esclarecida sob a forma de múltiplos fatores de risco envolvidos e que o tratamento deve ser realizado de forma multidisciplinar.

\section{Palavras-chave: Depressão Pós-Parto. Diagnóstico. Tratamento.}

ABSTRACT: This article highlights the thematic importance based on the focus on the population's mental health and how nurses contribute to recognition and functions through the early diagnosis of PPD as a way to alleviate the psychological distress of the parturient and the repercussions of psychological pain on social and family life. It reports the importance of prenatal care mediated by changes that can lead to postpartum depression. The present study seeks to clarify the etiology, the importance of early diagnosis, its various forms of treatment and preventive actions for postpartum depression, which result from

\footnotetext{
${ }^{1}$ Mestranda em Psicanálise com habilitação em Psicanálise Clínica - Supervisão Clínica SPSIG-Sociedade Psicanalítica Sigmund Freud de São Paulo - Instituto GAIO. Pós-graduação em Saúde Pública, Saúde Mental e Dependência Química- Faculdade de Ciências Humanas /ESUDA. Pós-graduação em Avaliação e Reabilitação Neuropsicológica e Neuropsicologia da Educação- Faculdade de Ciências Humanas/ ESUDA. Pós-graduação em Hematologia, Hemoterapia e Terapia de Suporte- Faculdade UNIBF. Pós-graduação em Análise do Comportamento Aplicada ao Autismo (ABA)- Faculdade UNIBF. Pós-graduação em Transtorno Borderline e Terapia Cognitivo-Comportamental - Faculdade UNIBF. Pós-graduação em Psicopedagogia Clínica e Institucional- Faculdade ALPHA. Pós- graduação em Educação Especial e Inclusiva- Faculdade ALPHA. Pós-graduação em Oncologia- Centro Universitário UNIFAVENI. Graduação em Licenciatura Plena em PEDAGOGIA com habilitação em Administração Escolar e Magistério - FUNESO/UNESF (Fundação de Ensino Superior de Olinda/ União de Escolas Superiores da FUNESO), Olinda-PE. Graduação em Bacharelado em Enfermagem (UNIBRA) - Centro Universitário Brasileiro, Recife-PE; andreazamoranozori@hotmail.com.
} 
emotional, cognitive, behavioral and physical changes triggered from the gestational onset to the puerperal stage. Through a bibliographical and systematic review of the literature, we observed that its etiology was not fully clarified in the form of risk factors involved and that the treatment should be carried out in a multidisciplinary way.

Keywords: Postpartum Depression. Diagnosis. Treatment.

\section{INTRODUÇÃO}

A Depressão Pós-Parto (DPP) é um quadro clínico severo e agudo que requer acompanhamento psicológico e psiquiátrico, pois devido à gravidade dos sintomas, há que se considerar o uso de medicação. Todo ciclo gravídico-puerperal é considerado período de risco para o psiquismo devido à intensidade da experiência vivida pela mulher. Esta experiência pode incidir sobre psiquismos mais ou menos estruturados. Mesmo mulheres com boa organização psíquica podem se ver frente a situações em que a rede social falha. Esta acomete entre 10\% e 20\% das mulheres, podendo começar na primeira semana após o parto e perdurar até dois anos.

A Depressão pós-parto (DPP), não afeta apenas a puérpera, mas todos os à sua volta, pode ser caracterizada como um transtorno no desempenho físico, comportamental, cognitivo e emocional, atingindo aproximadamente $15 \%$ das mulheres em geral, podendo ter início até 12 meses após o parto (ARRAIS; FRAGALLE; MOURÃO, 2014). Tendo diversos fatores de risco associados, dentre os quais alguns se destacam, tais como: histórico de algum transtorno psíquico, ter menos de 16 anos, baixa autoestima, solidão, gravidez não planejada, menor escolaridade, não estar casada em regime legal (solteira ou divorciada), abortamento espontâneo ou de repetição, desemprego da puérpera ou do companheiro, situações estressantes nos últimos 12 meses, ausência ou pouco amparo social, bebê do sexo oposto ao esperado, relacionamento insatisfatório (ALFAIA; MAGALHÃES; RODRIGUES 2016), a gravidez, parto (parto traumático, prematuro e histórico de aborto) e estresse (desemprego ou mortes), e por último a vida socioeconômica. Podendo observar se há histórico de depressão na família, e se mesma já apresentou algum episódio (ARRAIS; FRAGALLE; MOURÃO, 2014).

Os sintomas usualmente observados são: ansiedade, ideias de morte e suicídio, sentimento de culpa, diminuição do humor, diminuição ou perda do prazer, menor desempenho, distúrbio do sono (ALFAIA; MAGALHÃES; RODRIGUES 2016). 
Estudos apontam a possível relação entre o risco de depressão pós-parto com fatores relacionados anteriormente à tensão pré-menstrual. Essa relação é devido às semelhanças apresentadas em ambos os casos. Apresentando situações de alternância na esfera hormonal, tanto na queda dos níveis de progesterona como na função tireoidiana reduzida, as quais tem ligação direta com a qualidade de vida da mãe e do bebê (CABRAL; MARINI; MORAIS 2013).

Faz-se de extrema importância o acompanhamento pré-natal, pois segundo o Ministério da Saúde (MS) o foco desse acompanhamento é assegurar o desenvolvimento de uma gestação saudável com benefícios para a mãe e o bebê permitindo o nascimento de um bebê saudável e sem transtornos para a mãe em todos os seus aspectos, inclusive psicossocial (BOSKA; LENTSCK; WISNIEWSKI 2016).

Esse olhar cuidadoso do enfermeiro voltado a todas as gestantes, com medidas e ações de cuidado integral durante essa fase de mudanças e transições, poderá prevenir diversas complicações que podem ser provenientes da depressão pós-parto (ALFAIA; MAGALHÃES; RODRIGUES 2016).

Esta detecção deve ser feita no início de pós-parto ou então nas consultas pré-natais anteriores. Isto permite um tratamento adequado para as mães, para a relação mãe-recémnascido e, mais tarde, para o equilíbrio psicológico da criança. Portanto, o impacto na vida dos envolvidos requer um trabalho não só remediativo, mas também preventivo, a fim de evitar este grave transtorno. (ARRAES, ARAÚJO, SCHIAVO 2018).

A gravidez é um evento biologicamente natural, porém um período de importante vulnerabilidade emocional, onde sentimentos diversos são vividos, sendo essa fase uma transição que envolve a necessidade de reestruturação e reajustamento em várias dimensões. Causa mudança de identidade e redefinição de papéis. Mudanças iniciadas na descoberta da gravidez que se estendem além do parto, a depender da vivência de cada mulher (SAVIANIZEOTI; PETEAN, 2015).

Segundo Medeiros et al., (2016), mesmo se tratando de um fenômeno natural, deve ser acompanhada por uma equipe multidisciplinar, a fim de diminuir as intercorrências durante o pré-natal, trabalho de parto, parto e puerpério. Pois, uma assistência eficiente pode diminuir o surgimento de problemas reais e potências, facilitar a identificação dos diagnósticos, planejamento e implementação dos cuidados necessários. No pós-parto, a 
mulher pode apresentar maior risco para problemas de ordem física, mental e social. Atingindo também a família e a criança, sendo manifestados, muitas vezes, por: dores/incômodos; medos/preocupações; percalços sexuais; redução da autoestima e do cuidado pessoal; dificuldades interacionais com familiares e/ou com o filho; depressão e outros (TEIXEIRA et al., 2015).

Martinez et al., (2016), afirmam que durante o pós-parto, a condição psiquiátrica mais comum é a depressão pós-parto (DPP), um problema de saúde pública de escala mundial. E que apesar de uma proporção significativa das mães, enquanto usuárias da atenção primária de saúde (APS), apresentem para tal alto ENCICLOPÉDIA BIOSFERA, Centro Científico Conhecer - Goiânia, v.15 n.27; p. 2018, ainda sim os transtornos depressivos geralmente não são detectados ou tratados, apesar da disponibilidade de tratamentos eficazes. Este quadro manifesta-se, na maioria dos casos, a partir das primeiras quatro semanas após o parto e atinge, habitualmente, sua intensidade máxima nos seis primeiros meses (GALVÃO et al., 2015). Muitos fatores podem estar relacionados à DPP, como: histórico de depressão, transtorno mental, ansiedade ou problemas emocionais na gravidez, eventos estressantes, apoio social ou financeiro ausente ou insuficiente. E muitas são as consequências desse episódio, como o comprometimento da relação entre mãe e filho (BRITO et al., 2015).

Em mulheres com história prévia de depressão pós-parto (DPP), observa-se risco 70\% maior de desenvolverem outro episódio depressivo, e em casos de DPP prévia e melancolia da maternidade, baby blue, este risco aumenta para 85\%. (HARTMANN; MENDONZA-SASSI; CESAR, 2017).

Segundo a Organização Mundial de Saúde (OMS, 2017), a depressão é a principal causa de problemas de saúde e incapacidade em todo o mundo. É um importante fator de risco para o suicídio e é considerado um transtorno mental comum, caracterizada por tristeza persistente e uma perda de interesse e incapacidade de realizar atividades que a pessoa normalmente gosta e as atividades diárias por 14 dias.

Para o diagnóstico da DPP, a utilização de medicamentos antidepressivos tem se mostrado o primeiro recurso para o tratamento, embora ocasione prejuízos no aleitamento materno e poucas pesquisas tenham sido realizadas. Outro recurso, muito verificado que se mostrou eficaz foi a terapia cognitivo-comportamental - TCC (KROB, LEITE \& MORI, 2017). 
A Depressão pós-parto (DPP), não afeta apenas a puérpera, mas todos à sua volta, pode ser caracterizada como um transtorno no desempenho físico, comportamental, cognitivo e emocional, atingindo aproximadamente $15 \%$ das mulheres em geral, podendo ter início até 12 meses após o parto (ARRAIS; FRAGALLE; MOURÃO, 2014) tendo diversos fatores de risco associados, dentre os quais alguns se destacam, tais como: histórico de algum transtorno psíquico, ter menos de 16 anos, baixa autoestima, solidão, gravidez não planejada, menor escolaridade, não estar casada em regime legal (solteira ou divorciada), abortamento espontâneo ou de repetição, desemprego da puérpera ou do companheiro, situações estressantes nos últimos I2 meses, ausência ou pouco amparo social, bebê do sexo oposto ao esperado, relacionamento insatisfatório (ALFAIA; MAGALHÃES; RODRIGUES 2016), a gravidez, parto (parto traumático, prematuro e histórico de aborto) e estresse (desemprego ou mortes), e por último a vida socioeconômica. Podendo observar se há histórico de depressão na família, e se mesma já apresentou algum episódio (ARRAIS; FRAGALLE; MOURÃO, 2014).

Os sintomas usualmente observados são: ansiedade, ideias de morte e suicídio, sentimento de culpa, diminuição do humor, diminuição ou perda do prazer, menor desempenho, distúrbio do sono, (ALFAIA; MAGALHÃES; RODRIGUES 2016) descontrole emocional por estresse do parto, apresentando assim algumas disfunções como tristeza pós-parto, abatimento, choro repentino e incapacidade. Modificações do humor nas primeiras quatro semanas do puerpério, podendo ser leve e passageira ou piorar para uma neurose psicótica, sentimento de incapacidade, sono prejudicado, pensamento obsessivo, rejeição do bebê, riscos de suicídio ou infanticídio são características da doença (ARBOIT; DAANDELS; SAND, 2013).

Existem ainda fatores que podem sim ser predisponentes para o desenvolvimento de complicações e até mesmo uma depressão pós-parto. Podemos citar, por exemplo, o parto cesáreo, que tem sido realizado cada vez mais frequentemente e pode acarretar consigo transtornos de adaptação no pós-parto. A episiotomia, que segundo a OMS a indica em apenas $10 \%$ a $15 \%$ dos partos normais, no Brasil ela é realizada em aproximadamente $90 \%$ dos partos normais, o que ressalta seus agravos pois, pode trazer consequências não apenas físicas, mas também psicológicas à mulher no pós-parto (BOSKA; LENTSCK; WISNIEWSKI 2016). 
A ausência (ou não permissão) de um acompanhante durante o trabalho de parto e o momento do parto. A mulher, segundo a Lei do Acompanhante pode escolher alguém para acompanhá-la durante esse momento tão marcante em sua vida. Tendo consigo um acompanhante durante o trabalho de parto e o parto, segundo o Ministério da Saúde, a mulher se sentirá mais confiante e segura, o que possibilita a diminuição no uso de medicamentos analgésicos, a duração do trabalho de parto e até mesmo o número de partos cesáreas realizadas (BOSKA; LENTSCK; WISNIEWSKI 2016).

O programa de pré-natal psicológico é uma forma diferenciada dando assistência integral, trabalhando o processo gravídico-puerperal, complementando o pré-natal, onde trabalham vários aspectos importantes como modificações, confiança, vínculo com o bebê, cuidados na amamentação, tornando um local para expressar medos e ansiedades (ARRAIS; FRAGALLE; MOURÃO, 2014).

De que forma o enfermeiro poderá intervir para a prevenção à saúde mental da puérpera nos casos de depressão pós-parto e quais os benefícios do diagnóstico precoce de casos DPP acarretam a melhora do quadro e diminuição dos agravos à saúde mental da parturiente?

O profissional de saúde que assiste a parturiente deve conhecer e compreender os fatores socioculturais, ambientais, a fisiologia do processo do parto, fatores que possam causar medo e insegurança a essas parturientes, com a finalidade de proporcionar uma assistência humanizada e integral. Ressaltando a importância de identificar precocemente possíveis sintomas e fatores de risco, contribuindo assim, amenizar o sofrimento psíquico da parturiente e familiares através de tratamento medicamentoso e ações sociais e terapêuticas.

Buscamos analisar a depressão pós-parto a partir do enfoque à saúde mental da puérpera sob a perspectiva da enfermagem, a prevalência de fatores de risco e detecção precoce da sintomatologia referente à DPP entre as fases gestacional e puerperal; Investigar o papel do enfermeiro como corresponsável pelo acolhimento à puérpera promovendo subdiagnóstico; Ressaltar a importância do pré-natal e do apoio social e familiar como preventivo à DPP 


\section{DELINEAMENTO METODOLÓGICO}

Foi realizada uma revisão crítica das publicações na PUBMED, SCIELO e LILACS referidos à Depressão Pós-Parto. Foram excluídos os artigos que não atenderam aos critérios de inclusão, àqueles que apresentaram duplicidade de dados e os artigos que após leitura pormenorizada não atenderam ao objetivo proposto nessa revisão.

Trata-se de uma revisão bibliográfica da literatura do qual foi realizada busca em bases de dados eletrônicos, usando estratégias com os termos "Depressão Pós-Parto". Os critérios de inclusão foram aplicados aos registros obtidos em três etapas: títulos, resumos e textos completos de 25 artigos científicos publicados. Foram adotados como critérios de inclusão, artigos cujo título e/ou resumo sugerisse resultados originais sobre a prevalência ou incidência de DPP no presente estudo.

Foram excluídos 13 artigos em que faltavam informações sobre delineamento com foco apenas em adolescentes, HIV positivas ou pertencentes a minorias étnicas ou que não fosse possível distinguir resultados (Depressão Maior ou Menor) relativos a outros transtornos.

\section{REFERENCIAL TEÓRICO}

Foram identificados através dos resultados obtidos em pesquisa que utilizaram mulheres nos períodos de gravidez, pós-parto imediato e tardio, com ou sem histórico reprodutivo, dez fatores de risco considerados determinantes para o surgimento da depressão pós- parto, sendo eles: insatisfação com a gravidez; história de depressão; abuso sexual e violência doméstica antes, durante ou após a gravidez; maior número de estressores vivenciados ao longo da vida; história de depressão pós-parto; baixo índice de saúde mental; depressão pré-natal; sofrimento emocional durante a gravidez e/ou parto; aleitamento por menos de seis meses e baixo índice de ômega 3.

Gauthreaux et al. (2017), afirmou que a gravidez indesejada ou tardia está fortemente associado aos sintomas da DPP em relação às mulheres que desejavam engravidar. Para Turkcapar et al., revelou em suas pesquisas que mulheres com DPP era resultante da insatisfação com a gravidez atual. ENCICLOPÉDIA BIOSFERA, Centro Científico Conhecer - Goiânia, v.15 n.27; p. 2018. 
Segundo Gauthreaux et al. (2017), foi realizado um processo avaliativo através de questionários do qual esse achado foi obtido pelas mulheres categorizadas como gravidez indesejada ou tardia, o maior percentual por ter vivenciado em algum momento da sua vida um episódio depressivo. De acordo com Chojeta et al. (2016), a história de depressão se deve aos sintomas da DPP do qual o total de 5.219 mulheres que deram à luz,82o apresentaram sintomas depressivos pós-parto.

As evidências de Guathreaux et al. (2017) e Turkcapar et al. (E3), resultantes de questionários avaliativos aplicados às gestantes e parturientes, relataram que a gravidez indesejada ou tardia decorrente do abuso sexual e violência doméstica antes ou durante a gravidez, também considerando o seu histórico de reprodução, expressaram maior percentual de ocorrência de sintomatologia da DPP.

Para Gauthreaux e colaboradores (2017), foram considerados estressores: ter um membro da família doente; separação ou divórcio do parceiro; falta de moradia; a entrevistada ou o cônjuge perderam o emprego; conflitos com o parceiro; cônjuge não desejava gravidez; ser incapaz de pagar as contas; estar em uma luta física; a entrevistada ou o parceiro foram para a prisão; alguém perto da entrevistada usa drogas ou álcool ou alguém perto da entrevistada morreu. Foram achados para a amostra identificada como gravidez indesejada ou tardia, os seguintes resultados, quando: presença de I a 2 estressores aumentou a probabilidade de DPP pela metade; presença de 3 a 5 estressores - duplicou a chance de DPP e a presença de 6 ou mais estressores - quadruplicou a possibilidade dessas mulheres desenvolverem DPP. De acordo com Chojeta et al. (2016), foi realizado em seu processo avaliativo, os fatores de risco associados à DPP já vivenciados, tornando o fato episódico através de uma gestação atual comprometida com a DPP comparado às gestações anteriores, tornando-o recorrente. Esse achado fortalece o estudo de Turkcapar et al. (2015), que avalia mulheres que apresentaram sintomas da DPP atual, histórico de depressão pósparto, as quais demonstravam insatisfação com a atual gravidez e pensamentos suicidas em período gestacional.

Para avaliação de Chojeta et al. (2016), concluiu que as mulheres com sintomas de DPP apresentaram baixo índice de saúde mental, associados ao bem-estar físico e emocional dessas mulheres, ENCICLOPÉDIA BIOSFERA, Centro Científico Conhecer - Goiânia, 
V.15 n.27; p. 2018 Io também foi avaliado por esse questionário, sendo possível considerar a forte associação entre o baixo nível de saúde mental e a depressão pós-parto.

Existem evidências em todos os estudos analisados sobre a depressão pré-natal, porém somente Turkcapar et al. (2015), considerou em seus resultados que a depressão prénatal está relacionado a sintomatologia da DPP.

O sofrimento emocional durante a gravidez ou no parto foi demonstrado por Chojeta et al. (2016) e Markhus et al. (2013). Questionários aplicados expressaram relatos das puérperas sobre os eventos emocionais que envolveram a gestação e parto. Conclui-se que esse fator, quando associado a outros fatores de risco experimentados por elas, pode desenvolver um episódio de depressão no pós-parto.

Para Chojeta et al. (2016), este fator foi considerado um fator materno de risco para a DPP, após analisar no histórico reprodutivo e na gravidez atual, a desistência do aleitamento antes dos seis meses. Os estudos mostraram que o aleitamento por menos de seis meses afetou a relação da mãe com o bebê, diminuindo o vínculo materno. Concluindo que, quando associado a outros fatores de risco, podem interferir no bem-estar físico e mental da mulher, contribuindo para o surgimento de um episódio depressivo maior no puerpério (DPP).

Markhus et al (2013), realizou um estudo com 43 mulheres grávidas de 24 semanas, sugerindo que o baixo índice de ômega 3 no final da gestação, por volta de 28 semanas, vai interferir na saúde mental da gestante. Visto que, há uma grande demanda de ômega 3 para o feto e que a baixa reposição dietética ou mesmo a não reposição deste, pode comprometer o bem-estar mental dessa mãe e por consequência influenciar no surgimento da depressão pós-parto. Sua amostra foi avaliada através de questionários e de exames laboratoriais, onde conseguiu provar a forte associação entre o baixo nível de ômega 3 no fim da gestação e a presença do episódio depressivo no pós-parto.

Enfim, com base em dados coletados, a DPP é uma desordem psicológica que tem cura e que os profissionais acarretam o transtorno a uma formação acadêmica deficiente para se obter uma conduta protocolada na Atenção Primária, ressaltando a importância de um tratamento medicamentoso e psicoterapia com suporte para um apoio social e familiar. 


\section{RESULTADOS E DISCUSSÃO}

Caracterização dos artigos em análise. Recife, Pernambuco, 2019.

\begin{tabular}{|c|c|c|c|}
\hline $\begin{array}{l}\text { Autor / Ano de } \\
\text { Publicação }\end{array}$ & Título & Objetivo & $\begin{array}{l}\text { Síntese/ } \\
\text { Considerações }\end{array}$ \\
\hline $\begin{array}{l}\text { ARRAES, } \text { A. R.; } \\
\text { ARAÚJO, T.C.; } \\
\text { SCHIAVO, A.R. } \\
\text { 2018 }\end{array}$ & $\begin{array}{l}\text { Fatores de Risco e } \\
\text { Proteção Associados } \\
\text { à Depressão Pós- } \\
\text { Parto no Pré-Natal } \\
\text { Psicológico (PNP). }\end{array}$ & $\begin{array}{l}\text { Identificar fatores } \\
\text { de risco e de } \\
\text { proteção associados } \\
\text { à Depressão Pós- } \\
\text { Parto (DPP); a } \\
\text { avaliar } \\
\text { contribuição do Pré- } \\
\text { Natal Psicológico } \\
\text { (PNP) como } \\
\text { programa de } \\
\text { prevenção em Saúde } \\
\text { da Mulher. }\end{array}$ & $\begin{array}{l}\text { Entende-se que } \\
\text { fatores individuais e } \\
\text { subjetivos de cada } \\
\text { mulher impactam } \\
\text { diretamente à } \\
\text { vivência } \\
\text { maternidade. Ainda } \\
\text { são necessários mais } \\
\text { estudos para que } \\
\text { ampliem a expansão } \\
\text { da prática do PNP } \\
\text { para que não seja só } \\
\text { utilizada } \\
\text { contexto hospitalar } \\
\text { e sim durante essa } \\
\text { fase transitória da } \\
\text { vida. }\end{array}$ \\
\hline $\begin{array}{l}\text { ALFAIA, J.R.M., } \\
\text { RODRIGUES, } \\
\text { L.R., } \\
\text { MAGALHÃES, } \\
\text { 2oI6. }\end{array}$ & 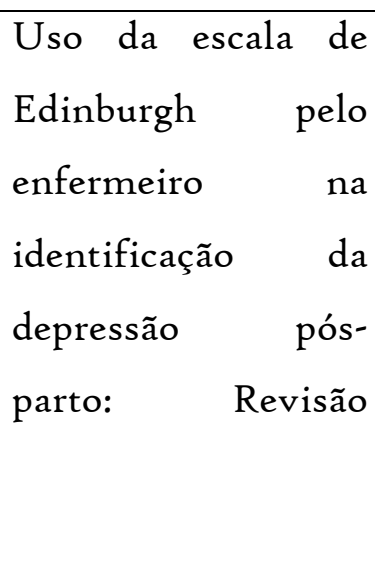 & $\begin{array}{l}\text { Analisar o uso da } \\
\text { Escala de Edinburgh } \\
\text { pelo enfermeiro na } \\
\text { identificação da } \\
\text { Depressão Pós- } \\
\text { Parto. }\end{array}$ & $\begin{array}{l}\text { É perceptível que o } \\
\text { infanticídio e o } \\
\text { suicídio estão entre } \\
\text { as complicações } \\
\text { mais graves } \\
\text { decorrentes da DPP, } \\
\text { sendo assim faz-se } \\
\text { necessário o }\end{array}$ \\
\hline
\end{tabular}




\begin{tabular}{|c|c|c|c|}
\hline & $\begin{array}{l}\text { Integrativa } \quad \mathrm{da} \\
\text { Literatura. }\end{array}$ & & $\begin{array}{l}\text { acompanhamento } \\
\text { cuidadoso de } \\
\text { gestantes. Além de } \\
\text { que o uso da escala } \\
\text { de Edinburgh é } \\
\text { extremamente } \\
\text { eficaz para o } \\
\text { diagnóstico de DPP. }\end{array}$ \\
\hline $\begin{array}{l}\text { BOSKA, G.A., } \\
\text { WISNIEWSKI, D., } \\
\text { LENTSCK, } \\
\text { M.H.,2оI6. }\end{array}$ & $\begin{array}{l}\text { Sintomas } \\
\text { depressivos no } \\
\text { período puerperal: }\end{array}$ & $\begin{array}{l}\text { Identificar sintomas } \\
\text { depressivos às } \\
\text { associá-los án } \\
\text { características } \\
\text { sociodemográficas e } \\
\text { clínicas de mulheres } \\
\text { no puerpério tardio. }\end{array}$ & $\begin{array}{l}\text { Ressalta-se a } \\
\text { importância de } \\
\text { acompanhar os } \\
\text { dados estatísticos } \\
\text { para associarmos à } \\
\text { evolução } \\
\text { diagnóstico } \\
\text { prevalente da DPP } \\
\text { em período } \\
\text { puerperal. }\end{array}$ \\
\hline $\begin{array}{l}\text { CHOJENTA CL, } \\
\text { Lucke JC, Forder } \\
\text { PM, Loxton DJ, } \\
\text { 2or6. }\end{array}$ & $\begin{array}{l}\text { Fatores de saúde } \\
\text { materna como riscos } \\
\text { para a depressão } \\
\text { pós-natal: um estudo } \\
\text { longitudinal } \\
\text { prospectivo. }\end{array}$ & $\begin{array}{l}\text { Explorar a relação } \\
\text { entre uma série de } \\
\text { fatores e o } \\
\text { construir } \\
\text { modelo } \\
\text { preditores de PND. }\end{array}$ & $\begin{array}{l}\text { É pertinente dizer } \\
\text { então, que a } \\
\text { compreensão do } \\
\text { histórico de saúde } \\
\text { mental de uma } \\
\text { mulher desempenha } \\
\text { um napel } \\
\text { importante na } \\
\text { detecção das pessoas } \\
\text { mais vulneráveis ao } \\
\text { PND. }\end{array}$ \\
\hline
\end{tabular}




\begin{tabular}{|c|c|c|c|}
\hline $\begin{array}{l}\text { DAANDELS, N., } \\
\text { ARBOIT, } \quad \text { E.L., } \\
\text { SAND I.C.P, 2013. }\end{array}$ & $\begin{array}{l}\text { Produção de } \\
\text { enfermagem sobre a } \\
\text { depressão pós- } \\
\text { parto. }\end{array}$ & \begin{tabular}{l}
\multicolumn{2}{l}{ Caracterizar estudos } \\
elaborados \\
enfermeiros \\
depressão \\
parto.
\end{tabular} & $\begin{array}{l}\text { Conclui-se que os } \\
\text { enfermeiros } \\
\text { estrangeiros têm- se } \\
\text { envolvido com o } \\
\text { aprofundamento } \\
\text { dessa temática há } \\
\text { cinco anos e que seus } \\
\text { estudos são mais } \\
\text { quantitativos. }\end{array}$ \\
\hline 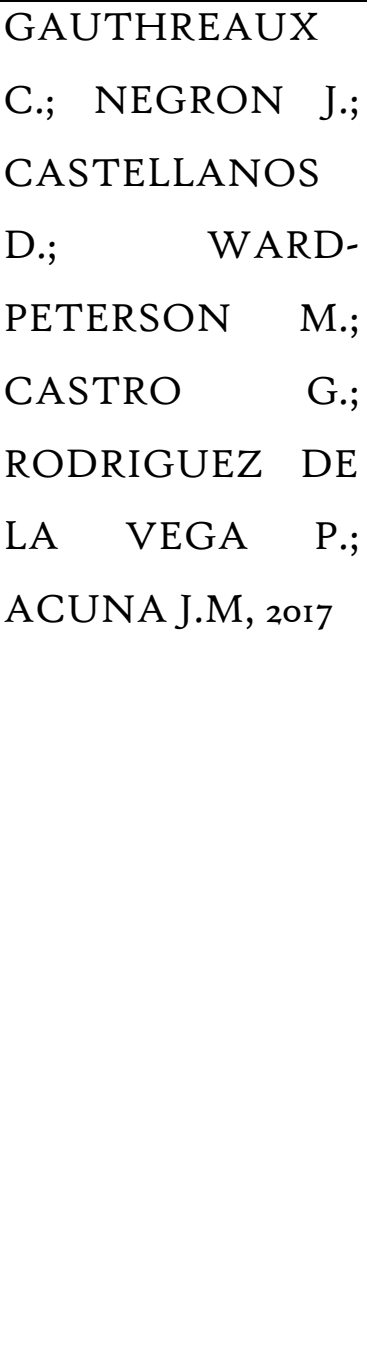 & $\begin{array}{l}\text { Associação entre a } \\
\text { adoção da gravidez e } \\
\text { a experimentação de } \\
\text { sintomas de } \\
\text { depressão pós-parto } \\
\text { entre novas mães } \\
\text { nos Estados Unidos. }\end{array}$ & $\begin{array}{l}\text { Verificar a } \\
\text { associação entre a } \\
\text { depressão pós-parto } \\
\text { e a ocorrência do } \\
\text { aleitamento } \\
\text { materno exclusivo. }\end{array}$ & $\begin{array}{l}\text { Infere-se que são } \\
\text { diversos fatores } \\
\text { associados dentre os } \\
\text { principais está a } \\
\text { importância de } \\
\text { estudos aue } \\
\text { investigue às } \\
\text { influência da saúde } \\
\text { mental da puérpera, } \\
\text { devido na } \\
\text { repercussões } \\
\text { causadas à saúde } \\
\text { interação mãe-bebêe } \\
\text { na prática do AME, } \\
\text { a fim de subsidiar } \\
\text { ações } \\
\text { promovam atenção } \\
\text { integral à } \\
\text { materno-infantil. }\end{array}$ \\
\hline
\end{tabular}




\begin{tabular}{|c|c|c|c|}
\hline $\begin{array}{l}\text { HARTMANN, M.J } \\
\text {; MENDONZA- } \\
\text { SASSI R. A, ; } \\
\text { CESAR, A. J. 2017. }\end{array}$ & $\begin{array}{l}\text { Depressão entre } \\
\text { puérperas: } \\
\text { prevalência e fatores } \\
\text { associados. }\end{array}$ & $\begin{array}{l}\text { Medir a prevalência } \\
\text { e identificar fatores } \\
\text { associados à } \\
\text { ocorrência de } \\
\text { depressão entre } \\
\text { puérperas residentes } \\
\text { em um município de } \\
\text { médio porte no } \\
\text { extremo Sul do país. }\end{array}$ & $\begin{array}{l}\text { Dessa forma, } \\
\text { entende-se os } \\
\text { fatores protetores } \\
\text { que se mostraram } \\
\text { significativamente } \\
\text { associados a essa } \\
\text { condição foram } \\
\text { suporte profissional } \\
\text { e apoio da equipe de } \\
\text { saúde durante o } \\
\text { parto, enquanto ser } \\
\text { adolescente, ter } \\
\text { maior paridade e } \\
\text { histórico de } \\
\text { depressão prévia ou } \\
\text { na família foram } \\
\text { considerados fatores } \\
\text { de risco. }\end{array}$ \\
\hline $\begin{array}{ll}\text { KROB, } & \text { D A; } \\
\text { GODO } & \text { Y, J.; } \\
\text { LEITE, } & \text { P. K; } \\
\text { MORI, } & \text { G. S; } \\
\text { 2017. } & \end{array}$ & $\begin{array}{l}\text { Depressão } \\
\text { Gestação e no Pós- } \\
\text { Parto e a } \\
\text { Responsividade } \\
\text { Materna nesse } \\
\text { contexto. }\end{array}$ & $\begin{array}{l}\text { Explanar a } \\
\text { responsividade } \\
\text { materna no contexto } \\
\text { da depressão pré- } \\
\text { natal e no pós-parto. }\end{array}$ & $\begin{array}{l}\text { Conclui-se que são } \\
\text { necessários } \\
\text { instrumentos e } \\
\text { intervenções } \\
\text { apropriadas para } \\
\text { essa patologia, sendo } \\
\text { essencial a avaliação } \\
\text { sobre a depressão } \\
\text { desde a gestação. } \\
\text { Além de que ainda é } \\
\text { um desafio para a } \\
\text { área da saúde o }\end{array}$ \\
\hline
\end{tabular}




\begin{tabular}{|c|c|c|c|}
\hline & & & $\begin{array}{l}\text { diagnóstico de DPP } \\
\text { realizado após o } \\
\text { nascimento do bebê. }\end{array}$ \\
\hline $\begin{array}{ll}\text { MORAIS, } & \text { E.A., } \\
\text { MARINI, } & \text { F.C., } \\
\text { CABRAL, } & \text { A.C., } \\
\text { 2013. } & \end{array}$ & $\begin{array}{l}\text { Associação entre } \\
\text { sintomas } \\
\text { emocionais da } \\
\text { tensão pré- } \\
\text { menstrual e o risco } \\
\text { de desenvolvimento } \\
\text { de sintomas } \\
\text { depressivos no pós- } \\
\text { parto. }\end{array}$ & $\begin{array}{l}\text { Verificar a possível } \\
\text { associação entre a } \\
\text { situação emocional } \\
\text { no pré-menstrual } \\
\text { (TPM emocional) e } \\
\text { risco de depressão } \\
\text { puerperal. }\end{array}$ & $\begin{array}{l}\text { Percebe-se que } \\
\text { existe sim uma } \\
\text { relação associativa } \\
\text { entre sintomas } \\
\text { emocionais no } \\
\text { período pré- } \\
\text { menstrual e a } \\
\text { ocorrência } \\
\text { de risco aumentado } \\
\text { para depressão } \\
\text { puerperal no } \\
\text { pós-parto imediato. }\end{array}$ \\
\hline $\begin{array}{lr}\text { MARKHUS } & \text { MW, } \\
\text { Skotheim } & \mathrm{S}, \\
\text { GRAFF } & \text { IE, } \\
\text { FROYLAND } & \mathrm{L}, \\
\text { BRAARUD } & \text { HC, } \\
\text { STORMARK } & \text { KM, } \\
\text { Malde MK. 2017 }\end{array}$ & $\begin{array}{l}\text { O índice de ômega-3 } \\
\text { baixo na gravidez é } \\
\text { um fator de risco } \\
\text { biológico para a } \\
\text { depressão pós-parto. }\end{array}$ & $\begin{array}{l}\text { Investigar se o baixo } \\
\text { índice de Omega-z é } \\
\text { um possível fator de } \\
\text { risco para a } \\
\text { depressão pós-parto. }\end{array}$ & $\begin{array}{l}\text { Conclui-se com base } \\
\text { nesses estudos que o } \\
\text { baixo índice de } \\
\text { ômega-3 está } \\
\text { associado com a } \\
\text { DPP. }\end{array}$ \\
\hline
\end{tabular}

\section{CONSIDERAÇÕES FINAIS}

Os estudos evidenciam que a DPP é um problema constante no cotidiano de profissionais da Atenção Básica do qual apresenta-se em posição favorável para detectar precocemente e intervir, evitando o agravamento da DPP do qual identificamos que sensibilidade materna é influenciada por fatores sócio-cognitivos e afetivos. A depressão pós-parto é reconhecida como um problema de grande incidência no Brasil. O diagnóstico 
precoce pode possibilitar a identificação da DPP e evitar que a mesma ocasione prejuízos à saúde da mãe e do bebê. Sabendo que o profissional da enfermagem é um dos responsáveis pelo direcionamento e orientação da gestante. Nesse sentido, é importante dizer que para identificar a depressão pós-parto é necessário um aprofundamento do histórico de vida da puérpera desde sua situação clínica e familiar. Para se chegar à conclusão desses argumentos, utilizou-se uma pesquisa de cunho bibliográfico e descritivo com coleta de dados em teses, artigos, livros e banco de dados eletrônicos que tratam da temática em questão.

A fase gestacional é caracterizada por uma transformação psíquica na mulher em razão das mudanças sociais, biológicas, culturais e históricas, ligadas ao estereótipo feminino. Apesar da gestação ser vista como um episódio normal na vida de uma mulher, exigem adaptações anatômicas, funcionais e emocionais. Para que a gravidez transcorra com segurança, são necessários cuidados a serem praticados pelas próprias gestantes, parceiro, família e especialmente pelos profissionais da rede de atenção básica de saúde. Nesse sentido, o papel da enfermagem dentro dessa perspectiva é que os profissionais têm um papel de grande importância na atuação da depressão pós-parto na intervenção eficaz no controle, prevenção e condução da gravidez na adolescência, tendo em vista a complexidade e especificidade assistencial desse tipo de público. O enfermeiro deve sempre agir como educador e os familiares como co-educadores para então, solucionar juntos, problemas de fundamentos comuns como a resistência e a desesperança. O profissional de saúde que assiste a parturiente deve conhecer e compreender os fatores socioculturais, ambientais, a fisiologia do processo do parto, fatores que possam causar medo e insegurança a essas parturientes, com a finalidade de proporcionar uma assistência humanizada e integral.

\section{REFERÊNCIAS}

ALFAIA, J.R.M., RODRIGUES, L.R., MAGALHÃES, M.M. Uso da escala de Edinburgh pelo enfermeiro na identificação da depressão Pós Parto: Revisão Integrativa da Literatura. Revista Ciência e Sociedade: vol. I, n. I, 20r6. Disponível em < http://revistaadmmade.estacio.br/index.php/cienciaesociedade/article/view/209I. 
ARRAIS, A.R., MOURÃO, M.A., FRAGALLE, B. O pré-natal psicológico como programa de prevenção à depressão pós-parto. Saúde Soc.: vol. 23, n. I, p. 251- 264, 2014. Disponível em < http://www.scielo.br/pdf/sausoc/v23nI/oro4-129o-sausoc-23-oI-0025I.pdf

BOSKA, G.A., WISNIEWSKI, D., LENTSCK, M.H. Sintomas depressivos no período puerperal: identificação pela escala de depressão pós-parto de Edinburgh. J Nurs Health: vol. I, n. $\mathrm{I}, \quad$ pag. 38-50, 20I6. Disponível < https://periodicos.ufpel.edu.br/ojs2/index.php/enfermagem/article/view/5525.

CHOJENTA CL, LUCKE JC, FORDER PM, LOXTON DJ.Fatores de saúde materna como riscos para a depressão pós-natal: um estudo longitudinal prospectivo. PLoS One. 20I6 Jan I9;II(I):eoI47246. doi: Io.137I/journal.pone.oI47246. eCollection 2016.

DAANDELS, N., ARBOIT, E.L., SAND I.C.P. Produção de enfermagem sobre a depressão pós parto. Cogitare Enfermagem: vol. 18, n. 4, p. 782-8, 2013. Disponível em: < http://revistas.ufpr.br/cogitare/article/view/34937 >

ENCICLOPÉDIA BIOSFERA, Centro Científico Conhecer- Goiânia, v.15 n.27; p.2018.

GAUTHREAUX C.; NEGRON J.; CASTELLANOS D.; WARD-PETERSON M.; CASTRO G.; RODRIGUEZ DE LA VEGA P.; ACUNA J.M. Associação entre a adoção da gravidez e a experimentação de sintomas de depressão pós-parto entre novas mães nos Estados Unidos. Medicine (Baltimore). 2017 Feb;96(6):e5851. doi: I0.1097/MD.0000000000005851.

HARTMANN, M.J ; MENDONZA-SASSI R. A, ; CESAR, A. J. Depressão entre puérperas: prevalência e fatores associados. Cadernos de Saúde Pública, or October 2017, Vol.33(9)

KROB, D A; GODOY, J.; LEITE, P. K; MORI, G. S. Depressão na Gestação e no PósParto e a Responsividade Materna Nesse Contexto. Revista Psicologia e Saúde, or September 2017, Vol.9(3), pp.3-16.

MARKHUS MW, Skotheim S, GRAFF IE, FROYLAND L, BRAARUD HC, STORMARK KM, Malde MK. O índice de ômega-z baixo na gravidez é um fator de risco 
biológico para a depressão pós-parto. PLoS One. 2013 Jul 3;8(7):e67617. doi: Io.1371/journal.pone.0067617. Print 2013.

MORAIS, E.A., MARINI, F.C., CABRAL, A.C.Associação entre sintomas emocionais da tensão pré-menstrual e o risco de desenvolvimento de sintomas depressivos no pós-parto. Rev Med Minas Gerais: vol. 23, n. 3, pag. 281-283,2013. Disponível em < http://rmmg.org/artigo/detalhes/208

TURKCAPAR, A.F; Kadığlu, N.; ASLAN, E.; TUNC, S.; Zayıfoğlu, M.;

Mollamahmutoğlu L. Sociodemographic and clinical features of postpartum et al 2015. 